Discussion The progressive increase in patients supported with this therapy mirrors its remarkable growth internationally. CVVH is the most dominant form of artificial renal support in Australian and European critical care, and its role as adjuvant therapy in sepsis is attracting increased focus. ${ }^{3}$

Conclusion CVVH activity is increasing at our institution, facilitated by a competent and flexible team of CVVH specialists. Future adequately powered multivariate logistic regression analysis should address outcomes of patients supported on CVVH.

\section{P0-0789 IMPACT OF HYPERCALCIURIA IN PAEDIATRIC RECURRENT URINARY TRACT INFECTION}

F Mortazavi, M Sheykhloo. Pediatric Nephrology, Tabriz University of Medical Sciences, Tabriz, Iran

\subsection{6/archdischild-2014-307384.1425}

Background Hypercalciuria has been considered as a predisposing factor for recurrent urinary tract infection (UTI) in recent studies. The mechanism may be related to uroepithelial injury by calcium microcrystals. The aim of this study was to evaluate the association of idiopathic Hypercalciuria with recurrent UTI in children.

Methods In this analytic study urine calcium:creatinine ratio of 40 children at the age of $2-16$ years with recurrent urinary tract infection (without urinary tract anomalies and voiding dysfunction) was compared with 40 age and sex matched healthy children. Calcium:creatinine ratio more than 0.2 in a spot urine test was considered as hypercalciuria. Cases with hypercalcemia were excluded from the study. Recurrent UTI was defined as at least 3 episodes of UTI during 1 year or 2 episodes in 6 months.

Results Mean age of patients was $5 \pm 2.22$ years and mean age of control group was $5.13 \pm 1.98$ years. The mean calcium:creatinine ratio in case group $(0.21 \pm 0.17)$ was significantly higher than control group $(0.08 \pm 0.08)(\mathrm{p}<0.05)$. Hypercalcuria was detected in nineteen out of forty patients in case group $(47.5 \%)$ and in $7.5 \%$ of control group ( $p<0.001)$. History of familial urolithiasis was positive in $21 \%$ of hypercalciuric patients. There was not any significant difference in frequency of urinary symptoms between hypercalciuric and normocalciuric patients with recurrent UTI.

Conclusion Children who suffer from recurrent UTI in spite of absence of urinary tract anomalies should be checked for hypercalciuria. Control of hypercalciuria with low salt regimen and high fluid intake and treatment with hydrochlorothiazide may decrease UTI episodes.

\section{P0-0790 FANCONI SYNDROME, VITAMIN D DEFICIENCY AND AKI IN BETA-THALASSEMIA PAEDIATRIC PATIENT RECEIVING DEFERASIROX: A CASE REPORT AND LITERATURE REVIEW}

${ }^{1} \mathrm{~N}$ Pravitsitthikul, ${ }^{2} \mathrm{~K}$ Torcharus. ${ }^{1}$ Pediatric Nephrology, Samitivej International Children's Hospital, Bangkok, Thailand; 'Pediatric Haemato-Oncology, Phramongkutklao College of Medicine, Bangkok, Thailand

\subsection{6/archdischild-2014-307384.1426}

Background and aims Deferasirox is a novel oral iron chelator for the treatment of iron-overload due to chronic hypertransfusion. Renal toxicity due to deferasirox was more recognised and deferasirox-induced tubulopathy has been increasing reports in the literature. We report a $\beta$-Thalassemia paediatric patient who developed Fanconi Syndrome (FS), AKI and vitamin D deficiency after deferasirox therapy.

Methods Patient's information was summarised and compared with literature.

Results A 4.8-year-old girl with $\beta$-Thalassemia major commenced hypertransfusion at 1.3 -year-olds and received deferasirox $21 \mathrm{mg} / \mathrm{kg} / \mathrm{day}$ at 1.9 -year-olds, which baseline serum ferritin was 2,216 ng/ml. After increasing deferasirox to $35 \mathrm{mg}$ / $\mathrm{kg} /$ day for 11 months, serum ferritin was lowering to $781 \mathrm{mg} /$ $\mathrm{ml}$. She was admitted with gastroenteritis, which revealed severe normal anion gap hyperchloremic hypokalaemic metabolic acidosis, severe hypophosphatemia, hypocalcemia, glucosuria, albuminuria, phosphaturia and vitamin D deficiency. Serum creatinine increased from $0.45 \mathrm{mg} / \mathrm{dl}$ to $0.75 \mathrm{mg} / \mathrm{dl}$ before turning to normal two months following cessation of deferasirox, as others except acidosis that persistently need alkali treatment. There were only five paediatric cases reported for deferasirox-induced FS in $\beta$-Thalassemia patients and 2 out of 5 that presented AKI. A prospective study in children reported 2 out of 10 cases presented deferasirox-induced FS, which 90\% were Thalassemia patients. Recovery of FS and AKI also literally presented within 3 months after deferasirox's cessation.

Conclusions - Deferasirox is potentially associated with renal toxicity in children, particularly FS and AKI.

- Diligent and regularly monitoring of renal function should be mandated in deferasirox receiving patients.

- Long-term consequences of kidney in deferasirox-treated children desire for further study.

\section{PO-0791 DIFFICULTIES IN DIAGNOSING VASCULITIS SYNDROME: A CASE REPORT OF A 10-YEAR-OLD BOY FROM MOLDOVA}

${ }^{1} \mathrm{~N}$ Revenco, ${ }^{1} \mathrm{O}$ Cirstea, ${ }^{2} \mathrm{D}$ Popusoi. ${ }^{1}$ Pediatrics, State Medical and Pharnaceutical University "Nicolae Testemitanu", Chisinau, Moldova; 'Student in General Medicine, State Medical and Pharnaceutical University "Nicolae Testemitanu", Chisinau, Moldova

\subsection{6/archdischild-2014-307384.1427}

Background Vasculitis presents several diagnostic challenges because patients could present with protean clinical manifestations and the range of differential diagnosis is broad. We report a pulmonary-renal syndrome in a 10 -year-old boy referred to the Institute for Maternal and Child Healthcare.

Materials and methods A 10-year-old boy presented in April 2013 with rhinitis, fever and dry cough. His examination results were unremarkable except mild anaemia $(9.9 \mathrm{~g} / \mathrm{dL})$ and markedly increased erythrocyte sedimentation rate $(44 \mathrm{~mm} / \mathrm{h})$. A month later he had been admitted with complaints of proteinuria, hematuria and anaemia. In June he developed also arthritis. In October 2014 the child was admitted to the Paediatric Intensive Care Unit in a severe condition with rapidly progressive renal failure.

Results and discussion Antineutrophil cytoplasmic antibodies (ANCA) were positive with antigen specificity for myeloperoxidase (anti-MPO $37 \mathrm{KU} / \mathrm{L}$ ). The other laboratory results included: mild anaemia and leukocytosis; proteinuria (69 mg/kg/day); increased blood urea nitrogen (BUN) and creatinine (10.4 mmol/ $\mathrm{L}$ and $123 \mathrm{mmol} / \mathrm{L}$, respectively). Thoracic CT revealed a solitary nodule $1.5 \times 1 \mathrm{~cm}$ in the posterio-basal segment of the inferior lobe in the left lung. The next step would be to perform renal biopsy to confirm the diagnosis.

Conclusions Our aim in presenting this case is to alert clinicians that, even without the definitive histological diagnosis, it is 